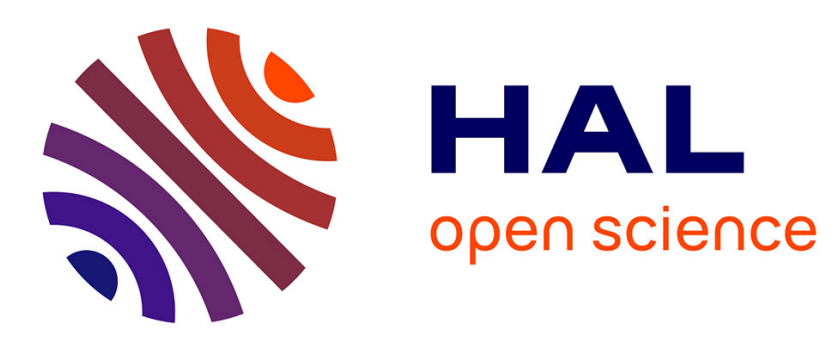

\title{
Artovarina : un théâtre résurrectionnel
}

Grossman Evelyne

\section{To cite this version:}

Grossman Evelyne. Artovarina: un théâtre résurrectionnel. Littérature, 2014, Valère Novarina: une poétique théologique?, 176, pp.88-96. halshs-01421456

\section{HAL Id: halshs-01421456 \\ https://shs.hal.science/halshs-01421456}

Submitted on 22 Dec 2016

HAL is a multi-disciplinary open access archive for the deposit and dissemination of scientific research documents, whether they are published or not. The documents may come from teaching and research institutions in France or abroad, or from public or private research centers.
L'archive ouverte pluridisciplinaire HAL, est destinée au dépôt et à la diffusion de documents scientifiques de niveau recherche, publiés ou non, émanant des établissements d'enseignement et de recherche français ou étrangers, des laboratoires publics ou privés. 


\section{Artovarina : un théâtre résurrectionnel \\ Evelyne Grossman}

« Du profond du théâtre, l'acteur nous apporte - dans sa bouche l'inhumanité du langage » (Valère Novarina, $L C$, p. 95)

[Résumé : La rencontre Novarina / Artaud eut lieu plus d'une fois. On ne racontera pas ici une histoire de famille, d'influence ou de filiation, mais une commune inquiétude - une interrogation partagée de l'inhumanité de la langue. Du coup de dés mallarméen aux anthropoglyphes-hiéroglyphes levés sur la page, se décèle une commune traversée de l'inhumain : Artovarina, corps-théâtre toujours en voie de surrection.

Sumary: The encounter between Novarina and Artaudtook placemore than once. Here wewill not tella tale of family, influence or parentage,but of commonconcern- a shared questioning of the inhumanityof language. From Mallarmé'scoup de dés to theanthropoglyphshieroglyphs raised onthe page, is being revealed a commoncrossing of theinhuman: Artovarina, a body-theatre always on the way of surrection.]

\section{Artovarina - Novarinarto}

Hasard objectif d'une rencontre, une autre, entreAntonin Artaud et Valère Novarina : juin 2010, colloque universitaire franco-espagnol organisé autour du recueilSuppôts et Suppliciations d'Antonin Artaud au très beau centre culturel, Arts Santa Mònica, de Barcelone. Au même moment était présentée dans la salle d'exposition du centre,une exposition de dessins de Valère Novarina, «Théâtre de dessins : 2587 personnages et 311 définitions de Dieu ${ }^{1} \gg$.Dans le catalogue de l'exposition, Brigitte Rambaud rappelle l'origine de cette performance (dans tous les sens du terme), entre pictographie et dramaturgie de l'extrême : les 5 et 6 juillet 1983, dans la grande salle de la tour Saint-Nicolas à La Rochelle, pendant vingt-quatre heures, Valère Novarinaexécuta à l'encre noire et au crayon rouge 2587 personnages. Elle raconte: "Le dessinateur se saisit d'un bambou et d'une feuille, les acteurs éparpillés à l'intérieur et à l'extérieur de la tour, dans des alcôves, sur le chemin de ronde, dans les escaliers commencent leur lecture des 2587 noms du Drame de la Viependant que Valère Novarina leur donne vie sur le papier. Je récupérerai toute la journée les dessins terminés et secs en attente sur sa table et les passerai aux accrocheurs ${ }^{2}$. »Le spectacle Le Drame de la Viesera créé en 1986 à Avignon.

Résonances croisées, donc, de scénographiescorporelles et psychiques: Artovarinaou Novarinartosur la scène de Barcelone.Dessinateurs et dramaturges, acteurs en langues plutôt qu'écrivains, chercheurs d'infini élevant une communeprotestation contre le carcan étriqué du corps humain, inventantun nouveau langage au plus près des rythmes corporels et vocaux :

${ }^{1}$ Le catalogue en français et catalan, précédé d'une préface de VicençAltaió, fut publié sous le même titre : Valère Novarina, Teatre de dibuixos : 2587 personatges i 311 definicions de Déu, Arts Santa Mònica, Eumo Editorial, 2010.[désormais abrégé en Catalogue Arts Santa Mònica]

${ }^{2}$ Brigitte Rambaud, « Deux mille cinq cent quatre-vingt-sept », Catalogue Arts Santa Mònica, op. cit., p. 6. 
Faire des livres, ça ne m'intéresse pas. C'est l'expérience, l'épreuve, l'exercice que je cherche. [...] Mais ce n'est pas du tout de la littérature de recherche, de laboratoire, [...] mais une expérience biologique sur soi, une intervention sur notre corps vivant [...]. J'entends la matière comique, $j$ 'entends les langues circuler autrement, $j$ 'invente un corps nouveau, je détruis mon image d'homme, je deviens un corps de langue, un comique mutant ${ }^{3} \ldots$ »

Valère Novarina rappelle volontiers qu'il a autrefois écrit sur Antonin Artaud: « 1964 : Etudes de Lettres, à la Sorbonne. Ecrit un mémoire sur Antonin Artaud, théoricien du théâtre. ». Le mémoire, Diplôme d'Etudes Supérieures soutenu à l'Institut d'Etudes Théâtrales de l'ancienne Sorbonne, est toujours répertorié à la Bibliothèque Gaston Baty de l'université Paris 3.En 1964, beaucoup de textes d'Artaud étaient encore dispersés dans des revues, et singulièrement ceux rédigés durant ses séjoursasilaires entre 1937 et 1946. Le premier tome de ses Euvres complètes n'était paru chez Gallimard qu'en 1956, après bien des aléas et querelles post mortem avec les héritiers. En 1964, Paule Thévenin, la première éditrice des œuvres, faisait paraître le tome V («Autour du Théâtre et son double et des Cenci »). Plus d'une vingtaine d'autres volumes suivraient jusqu'en 1994; Suppôts et Suppliciations,recueil posthume patiemment recomposé par Paule Thévenin, ne parut qu'en 1978. La plupart des grands textes d'Artaud sur le théâtre étaient donc déjà rassemblés à l'époque où le jeune Novarina travaille sur l'œuvre mais les derniers écrits sur le «nouveau théâtre de la cruauté » et les commentaires des dessinsn'étaient pas aisés à trouver, si ce n'est en bibliothèque. Il y travailla beaucoup.

\section{Qu'y a-t-il dans un nom...?}

Valère Novarina dit n'être plus,depuis lors,revenu à Artaud mais Artaud revient çà et là dans l'œuvre de Novarina, en légers signes complices sans qu'il soit besoin d'autre preuve de proximité. Ceci n'est pas une histoire de famille, d'influence ou de filiation, mais de commune inquiétude - une interrogation partagée de l'inhumanité de la langue.

Des citations, des allusions à Artaud, on peut s'amuser à en relever quelques-unes dans les textes de Novarina. Par exemple ceci,presque au hasard :

- Dans L'Acte inconnu, la « Machine à faire l'homme » évoque « Sotteville-lès-Rouen » $\left(A I^{2}\right.$, p. 91), nom de la ville où se trouvait l'hôpital psychiatrique départemental des Quatre-Mares dans lequel Artaud fut interné sous placement d'office en octobre 1937 à la suite de son séjour en Irlande. Il y adresse la seule lettre de lui connue de ses premiers enfermements, «au ministre plénipotentiaire d'Irlande à Paris », lui réclamant sa libération immédiate. Début d'une longue série d'enfermements asilaires qui vont durer 9 ans.Plus loin, à nouveau la « Machine à faire l'homme »fait allusion à une « brillante consœur des Nouvelles Révélations de l'Être » $\left(A I^{2}\right.$, p. 118). Titre parodiqued'un journal imaginaire, du type «Les Dernières Nouvelles d'Alsace » ou «La Nouvelle République des Pyrénées »,certes, mais Les Nouvelles Révélations de l'Être estaussi comme l'on sait le titre d'une étrange plaquette d'Artaud, imprimée en 1937quelques mois avant sa plongée dans la psychose et qu'il signa «Le Révélé », effaçant ainsi son nom.

- Dans Le Discours aux animaux, alors que l'homme est sommé de dire son nom («Dis ton nom, crâne sans nom!»), s'élève une longue litanie des mi-hommes mi-animaux, « omnimaux », « omniriens », « amnimaux »hors noms, hors lignée : «Jean des Réseaux des

3 «Le théâtre séparé », Réponse deValère Novarina à quatre questions de Philippe di Meo, Furor $\mathrm{n}^{\circ} 5$, Lausanne, janvier 1982. 
animaux,[...]son chien par alliance, son cheval par les femmes, Jean du Tombe Long, [...] Jean Paul de Bref, mon fils mon père ma mère et moi qui sortent habillés d'terre et teints en vrac » (DA, p. 316). On reconnaît au passage le début de Ci-Gît : «Moi, Antonin Artaud, je suis mon fils, mon père, ma mère, et moi ; niveleur du périple imbécile où s'enferre l'engendrement, le périple papa-maman et l'enfant... ». C'est dire que l'homme animal n'entre pas si tranquillement dans une généalogie linéaire ; « ... j'ai senti, moi, à quel point je ne supportais pas le végétal, ni le minéral, ni l'animal, ni l'ominal ${ }^{4}$ », écrit aussi Artaud. Enoncé psychotique ? Ou plutôt, comme l'énonce Novarina, "s'affranchir de l'identité humaine » $\left(D P^{2}\right.$, p. 44) ? L'acteur, chez Novarina, « transhomme ».

\section{Expulsion - projection}

A propos de sa pièce Le Vrai sang, Valère Novarina évoque un «théâtre de carnaval, en ce sens que les acteurs à la fois incarnent et quittent la chair, sortent d'homme, deviennent des figures qui passent sur les murs, des animaux peints, des signaux humains disséminés dans l'espace [...]. Le langage vient ici nous ouvrir, opérer devant nous le théâtre de la cruauté comique 5 . »Le théâtre de la cruauté,comme il le souligne à juste titre,c'est en effet des signes disséminés dans l'espace: «machine de souffle», dit Artaud, poésie-force, incantation, rythme, "poésie dans l'espace » (c'est cela qu'Artaud appelle théâtre et qui revivra sur les pages de ses cahiers d'écolier à la fin de sa vie), mouvement des syllabes proférées, expectorées - corps animé des mots.

De l'un à l'autre, on pourrait ainsi tisser les échos d'une recherche obstinément réitérée. «Théâtralité respiratoire de la page », écrit Novarina $\left(T P^{2}\right.$, p. 140$)$; «musculature affective » des souffles, «répétitions rythmiques de syllabes », renchérit Artaud. «Cruauté articulatoire, carnage langagier », reprend $\operatorname{Novarina}\left(T P^{2}\right.$, p. 12); 'acteurdoit travailler son corps «dans l'centre [...]; Dans les muscles du ventre. [...] Là d'où s'expulse la langue qui sort, dans l'endroit d'éjection, dans l'endroit d'l'expulsion d'la parole, là d'où elle secoue le corps entier $\gg\left(T P^{2}\right.$, p. 23$)$.

Expulsion - éjection, redit Novarina.

Déjection - projection répète Artaud

Ce que cherche Artaud, il le souligne dans ses derniers textes, c'est à incarner dans son corps d'écriture le corps infini de la langue. C'est du corps mortel qu'il faut sans relâche sortir, pour dresser, dans le théâtre de l'écriture, la scène infiniment reprise où se refait le corps de langue, « un corps en érection, en état d'éjection, de trépidation perpétuelle ${ }^{6}$ ». Que signifie être «en état d'éjection », sinon être ce suspens tendu, cette tension entre éjection et déjection. Je m'éjecte constamment de mon corps d'homme, ce corps voué à la mort, ce corps déchet, jeté à la naissance, et je fais du déchet, de la déjection, un projectile, un corps en éjection, en perpétuelle sortie de lui-même.Nous défaire de l'homme, dit Novarina, le mettre «hors de lui » ( $L C$, p. 78). Sortie de soi donc, ou comme le formule Mallarmé, "souci d'extravaguer du corps ${ }^{7} \gg$.

\footnotetext{
${ }^{4}$ Antonin Artaud, Suppôts et Supplications, Euvres, Quarto-Gallimard, 2004, p. 1412. Désormais noté $Q$, suivi de la page.

${ }^{5}$ Le Vrai sang, feuille de salle à l'Odéon-Théâtre de l'Europe, janvier 2011.

${ }^{6}$ « Histoire vécue d'Artaud Momo », Euvres complètes, édition de Paule Thévenin, tome XXVI, p. 158.

${ }^{7}$ Stéphane Mallarmé, "Quant au livre »,Euvres complètes, Gallimard, Bibliothèque de la Pléiade, éd. H Mondor, p. 369.
} 


\section{La scène du coup de dés}

A l'origine, toujours, chez l'un et l'autre, Mallarmé : «A l'âge de 18 ans, déclare Novarina dans un entretien, j'ai eu une sorte de bizarre illumination en feuilletant dans tous les sens une grosse thèse très mystérieuse écrite sur Un coup de dés de Mallarmé [...] soudain, dans la bibliothèque Sainte-Geneviève, je me suis cru placé à la croisée du drame du corps et de la parole. Au croisement des contraires : la page plate du livre et la page charnelle du théâtre, en volume. »C'est ainsi que sur la scène de théâtre les acteurstracent dans l'air des lettres, émettent des signes; les mots sont des trajets, des souffles : "L'acteur lance ses anthropoglyphes, émet et sème des lettres dans l'espace ${ }^{8} »$. Et de même, chez Artaud, les acteurs du théâtre balinais, modèles du théâtre dont il rêve, sont des « hiéroglyphes animés »:

«Cet espace d'air intellectuel, ce jeu psychique, ce silence pétri de pensées qui existe entre les membres d'une phrase écrite, ici, est tracé dans l'air scénique, entre les membres, l'air, et les perspectives d'un certain nombre de cris, de couleurs et de mouvements. » $(\mathrm{Q}, 541)$

Que les mots donc, redeviennent vivants et vibrants, matière sonore et visuelle, qu'ils se déploient comme des gestes physiques et concrets dans toutes les dimensions de la scène : idéogrammes, hiéroglyphes, anthropoglyphes. Alors la diction que l'acteur imprime au langage proféré, projeté sur la scène, se mue en force répétitive qui déstabilise l'ordre linéaire : vibrations, échos de bruits, modulation des voix, force de projection des syllabes dans l'air, mise en acte d'une parole-matière, indistinctement visuelle et sonore.

\section{Résurrection - Insurrection}

Mallarmé, toujours. Dans un article intitulé « Notre parole », publié dans le journal Libération en 1988, Novarina reprenait la métaphore mallarméenne récusant l'échange des mots comme échange de monnaie ${ }^{9}$ : «Parler n'est pas échanger des choses, communiquer des mots, sonnants et trébuchants... »Bien au contraire, souligne-t-il : «Celui qui parle ne s'exprime pas, il renaît. Parler respire et la pensée délie. Toute vraie parole est résurrectionnelle » $\left(T P^{2}\right.$, p. 233).

On sait que Novarina est un lecteur des Pères de l'Eglise, eux qui précisément postulèrent dans l'être humain un double corps, l'un mortel voué à la putréfaction, et l'autre inorganique, spirituel et glorieux, promis à la résurrection. Ainsi saint Augustin, dans le dernier livre de la Cité de Dieu,explore avec une minutieuse gravité la forme que prendra après la mort le nouveau corps spirituel : «Si les enfants ressusciteront avec le même corps qu'ils avaient à l'âge où ils sont morts » (chapitre XIV) ; "Si la taille de Jésus-Christ sera le modèle de la taille de tous les hommes, lors de la résurrection » (chapitre XV); "Si les femmes, en ressuscitant, garderont leur sexe » (chapitre XVII).

C'est à ce corps «glorieux» («sempiternel », dit Artaud) que bien des écrivains et penseurs modernes confrontent leur corps d'écriture. De Blanchot à Derrida, de Deleuze à Beckett, peu d'entre eux pourtant l'ont fait de façon aussi obstinée et rigoureuse que Valère Novarina, lui qui affirme qu'il n'y a pas de différence entre la linguistique et la théologie (...

\footnotetext{
${ }^{8}$ Valère Novarina, «L'homme hors de lui », entretien avec Jean-Marie Thomasseau, revue Europe, $\mathrm{n}^{\circ}$ 880-881, août-septembre 2002, respectivement p. 166 et 171.

${ }^{9}$ Mallarmé, on s'en souvient, critique cet emploi élémentaire du discours qui consisterait « à mettre ou à prendre dans la main d'autrui en silence une pièce de monnaie ». Stéphane Mallarmé, « Crise de vers », op. cit., p. 368.
} 
ou Artaud, lui qui, du fond de sa psychose lumineuse, déclare finalement : «Dieu, de son vrai nom, s'appelle Antonin Artaud »).

Sans cesse, donc, ouvrir l'homme; car la question n'est guère de savoir si l'un et l'autre croient en cette résurrection chrétienne des corps. Sans doute pas plus que nous, «athées névrosés », comme dit Novarina.Bien plutôt, ils en explorent inlassablement la logique, en appellent à l'insurrection de la lettre, à sa surrection, à sa levée ... en corps.

"L'âme est un suppôt, écrit Artaud, non un dépôt mais un suppôt, ce qui toujours se relève et se soulève de ce qui d'autrefois a voulu subsister, je voudrais dire rémaner, demeurer pour réémaner, émaner en gardant tout son reste, être le reste qui va remonter" (Q, 1061). Le suppôtest soulèvement de l'être, insurrection du corps, l'inverse de la chute des corps humains dans le monde phénoménal des organismes séparés. Seule la force de l'écriture théâtrale en acte, qui sans fin relance le mouvement des lettres-corpuscules peut empêcher le corps de s'anatomiser.

Et Novarina élève un culte à l'acteur Louis de Funès, «figure de la transfiguration comique »: «L'acteur est aujourd'hui, plus que tout humanologue, programmaliste, sociologueur, recteur légiste, celui qui en sait le plus sur la pratique mentale pure, [...]la combustion du corps et de l'esprit, la renaissance psychique, le rêve et les records de résurrection, sur la chute, la gloire, la rechute $[\ldots] »\left(T P^{2}\right.$, p. 165).

Chez eux, comme chez Beckett, le clown-acteur, Wladimir-Estragon, choit et se relève... Le début de la surrection, c'est la chute : chute des corps, re-surrection ${ }^{10}$.Non plus seulement donc la résurrection des corps (version christique ou théologique) mais le soulèvement de l'être, l'insurrectiondu «corps animé » des lettres comme force et projection de souffle, explosion de matière. «La force du corps lui-même, dit Artaud, latent en train de s'élever / [...] l'épaisseur du corps en tapulte, en catapulte projeté...» (Suppôts et Suppliciations)

«Parce que les tombes des acteurs sont profondes très peu. Un simple rideau de terre leur suffit. Pour qu'ils soient les premiers à toujours resurgir. Avec leurs corps légers, libres, volcaniens » $\left(T P^{2}\right.$, p. 204).

Artovarina, corps-théâtre toujours en voie de surrection. «Le théâtre doit devenir le lieu d'un lyrisme sans moi. Le je y est un assemblage », écrit Novarina ( $L C$, p. 32)

\section{De l'anti-humanisme}

Lorsque Lyotard publie L'inhumain en 1988, cela fait bien longtempsque la notion d'humanisme a été remise en question, historicisée. En France, c'est dans les années soixante que se dessine ce qu'on appellera plus tard la querelle de l'humanisme autour du structuralisme (Lévi-Strauss), de la psychanalyse (Lacan), de la sémiologie (Barthes), de la philosophie (Foucault, Althusser et d'autres...) de la littérature (le Nouveau Roman, puis Blanchot, Beckett,entre autres). Dans un entretien de 1966 intitulé «L'homme est-il mort ?», Michel Foucault résume ce qu'il développe dans Les Mots et les choses, à savoir ceci : l'humanisme est un mirage, une illusion rétrospective de nos cultures occidentales : "Nous

\footnotetext{
${ }^{10}$ Version plus sérieuse, mais c'est la même,dansLumières $d u$ corps : « Le texte revient de la mort.En grec

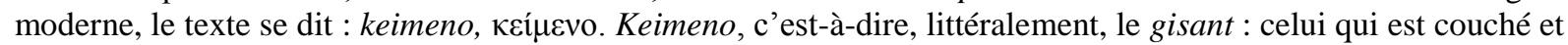
que l'acteur relève, ce qui est mort et que l'acteur ressuscite. L'acteur est un homme debout qui relève celui qui gisait. Il change les lettres en parole. Par le corps de l'acteur, la lettre vit; par le don du souffle, le texte ressuscite. Seul l'acteur, par son souffle, son offrande respiratoire - par son pouvoir d'inversion et de renversement -, fait que le texte se relève et tient debout $\gg(L C$, p. 107).
} 
imaginons que l'humanisme a été la grande force qui animait notre développement historique et qu'il est finalement la récompense de ce développement [...]. Ce qui nous émerveille dans notre culture actuelle, c'est qu'elle puisse avoir le souci de l'humain. Et si l'on parle de barbarie contemporaine, c'est dans la mesure où les machines, ou certaines institutions nous apparaissent comme non humaines ${ }^{11_{1}}$.

Or tout cela, précise Foucault, n'est pas exact. Le mouvement humaniste date de la fin du XIXème siècle. C'est parce qu'on a construit l'être humain comme objet d'un savoir possible que se sont ensuite développés tous les thèmes moraux de l'humanisme contemporain, et en particulier, ces « humanismes mous » que furent en France Camus, SaintExupéry ou Teilhard de Chardin. En fait, conclut-il, «l'homme est une invention dont l'archéologie de notre pensée montre aisément la date récente. Et peut-être la fin prochaine ». On connaît la métaphore célèbre du visage humain s'effaçant sur le sable...Que l'humanisme ait été, dès l'origine, une théologie inversée mettant l'homme à la place de Dieu, bien des philosophes le diront. Foucault insiste : la culture nouvelle qui apparaît au XXème siècle a commencé avec Nietzsche, lorsqu'il a montré que la mort de Dieu n'était pas l'apparition mais la disparition de l'homme, que Dieu étant mort l'homme n'a pas pu ne pas disparaitre en même temps.

Cette question de la mort, avec celle de Dieu, d'un certain humanisme occidental et sa vision d'un sujet centré, intentionnel et conscient, comme le dit Derrida, est au cœur, comme l'on sait, de bien des écritures littéraires et philosophiques du XXème siècle. De là sans doute, leur exploration constante et méthodique, de l'inhumain. L'hypothèse que reprend Lyotard n'est pas nouvelle : le «propre » de l'homme est qu'il est habité par de l'inhumain ; il est hanté par un hôte familier qui le fait délirer mais aussi penser ${ }^{12}$.

Au-delà même de ce que suggère Lyotard, je verrais volontiers dans cette exploration contemporaine de la mystique, qu'elle avoue ou non son nom (je pense à Blanchot, Bataille, Artaud, Derrida, Deleuze, Duras, Michaux, Merleau-Ponty, Novarina, etc.), un désir de réinventerl'inhumanité de l'homme: ce qui "passe infiniment l'homme», comme disait Pascal, la sortie des limites humaines ; à entendre aussi bien comme la part d'animalité que comme la part de divin, le risque de la folie, de la démesure, de la barbarie.La question actuelle pourrait alors être celle-ci : comment, à partir de la mort de cette figure traditionnelle de l'homme (celle, des humanismes «mous » de Foucault, qui n'ont guère su résister aux vagues déferlantes des barbaries des XXème et XXIème siècles), comment réinventer un autre humanisme qui prenne en compte l'inhumain, qui ne le refoule pas, ne les dénie pas, mais en inclue les potentialités terrifiantes, afin de les affronter en connaissance de cause?

Question annexe: pourquoi rencontrons-nous au cœur de cette interrogation une étonnante proximitédes écrivains et des philosophes ? Je ferais l'hypothèse que c'est parce qu'il y a eu au XXème siècleune véritable divinisation du langage. Ainsi, lisant Blanchot, Foucault affirme-t-il que seule la littérature, à cause de l'expérience de l'infini dulangage qui est la sienne, son expérience de l'être du langage, peut constituer, une « expérience de pensée radicale $^{13} »$.

Or qu'est-ce que le langage désormais ? Non plus «le propre de l'homme », ce qui le sépare irréductiblement des animaux, pour ne rien dire des pierres, comme le répétait le discours de l'humanisme classique... Tout au contraire, dirais-je, la découverte du XXème siècle, c'est que le langage est l'essence inhumaine del'homme, ce qui le divise et le rend autre à lui-même (Freud, Lacan), ce qu'il ne possèdera jamais «en propre », auquel il

\footnotetext{
${ }^{11}$ Dits et Ecrits[1994], Quarto-Gallimard, tome 1, 2001, p. 568.

12Jean-François Lyotard, L’inhumain. Causeries sur le temps, Galilée, 1988, p. 9-15

${ }^{13}$ Michel Foucault, «La pensée du dehors » [1966], Dits et EcritsI, op. cit., p. 546-567.
} 
demeure toujours étranger (Derrida, Deleuze), cet infini dont l'éternel murmure menace de le rendre fou (Blanchot, Levinas, Artaud...).

\section{L'ahomme ' $^{14}$}

Valère Novarina, le répète : «Les personnages de La Scène, de L'Origine rouge, de L'Espace furieux, de L'Opérette imaginaire, ne sont pas des hommes mais des animaux devant nous qui émettent des signaux humains. [...] L'art de l'acteur est un inhumanisme militant pratiqué au grand jour» $(L C$, p. 28). Dans l'entretien avec Jean-Marie Thomasseauque je citais plus haut, à la question « le novarinisme est-il un humanisme ?», il répond ceci : «Pas du tout. [...] Humaniste non. Je sors d'homme. [...] Il faut sur les planches, fabriquer une antipersonne, un anti-homme expérimental. Pousser l'homme hors de lui, lui faire porter son langage dehors. L'homme soudain furieux, forcené, jeté hors du langage. C'est un exercice exanthropique ${ }^{15}$. »

On connaît la condamnation par Artaud de l'humanisme occidental. Sans relâche il interroge les systèmes symboliques, religieux ou philosophiques dans lesquels l'individu prend sens de se dissoudre dans une subjectivité qui l'absorbe et le dépasse. Il écrit par exemple ceci dans une lettre : «[...] ce que les hommes appellent aujourd'hui l'humain, c'est le châtrage de la partie surhumaine de l'homme. » L'homme selon Artaud, c'est cette part d'inhumain qui nous rattache à l'illimité, c'est la folie que la rationalité occidentale a châtrée. C'est précisémentcet «homme psychologique » que le théâtre doit dissoudre afin de retrouver les véritables dimensions du corps et de la langue : sans limites. Dans un texte rédigé en commentaire de l'un de ses dessins, Le visage humain, il écrit ceci : " J'ai fait venir parfois, à côté des têtes humaines, des objets, des arbres ou des animaux parce que je ne suis pas encore sûr des limites auxquelles le corps du moi humain peut s'arrêter » $(Q, 1535)$.

En ce sens, et pour reprendre une formule de Gilles Deleuze, l'homme en fin de compte n'aura peut-être été qu'une manière d'emprisonner la vie. Une autre forme sans doute est en train de naître, qui n'est pas nécessairement une forme humaine : « ce pourra être une forme animale dont l'homme sera seulement un avatar, une forme divine dont il sera le reflet [...] Aujourd'hui l'homme entre en rapport avec d'autres forces encore (le cosmos dans l'espace, les particules dans la matière, le silicium dans la machine...) : une nouvelle forme en naît, qui n'est déjà plus celle de l'homme ${ }^{16}$ ». En d'autres termes : humain versus inhumain, ce ne sera plus vraiment la question mais plutôt celle d'une puissance de vie non organique dont l'homme n'aura été qu'une forme provisoire.

«L'homme, dit Valère Novarina, il faut le représenter à nouveau troué par Dieu ou en animal. Ou muet comme les pierres » $(L C$, p. 77$)$.

\footnotetext{
${ }^{14}$ «L'acteur est le décompositeur del'homme. [...] Il représente l'homme arraché à lui, enlevé d'ici, décorporé, défait, antipersonne; il représente l'homme asomatique, apathique, et en antipsychie. L'ahomme. » (LC, 150151)

${ }^{15}$ « L'homme hors delui », op. cit., p . 173.

${ }^{16}$ Gilles Deleuze, entretien avec Claire Parnet, Pourparlers, Minuit, p. 159-160.
} 